\title{
A pandemic on the horizon
}

\author{
Liam Donaldson
}

J R Soc Med 2006;99:222-225

This essay is based upon a presentation delivered at The Royal Society of Medicine by Sir Liam Donaldson, Chief Medical Officer, on 18 January 2006. The figures contained herein are up-to-date as of 11 April 2006.

\section{SUMMARY}

A pandemic of human influenza is on the horizon: the question is not whether it will arrive but when. Knowing that a pandemic is coming provides an opportunity to plan ahead to mitigate against its impact. However, there are inevitably many uncertainties and managing these, under a constant media spotlight, is an additional and novel challenge.

\section{INTRODUCTION}

The influenza virus is a well established public enemy. The three types of virus (A, B and C) can infect humans while type $A$ is also able to cause disease in birds, pigs and other animals. Prominent infectious diseases have long been reflected in nursery rhymes: ring a ring o' roses is said to have arisen at the time of the Great Plague. In 1918, at the time of the Spanish flu pandemic, which resulted in 15000 excess deaths each week in England and Wales, another rhyme became popular amongst children on British streets:

$$
\begin{aligned}
& \text { I had a birdy, } \\
& \text { His name was Enza, } \\
& \text { I opened the window, } \\
& \text { and in flew Enza . . . }
\end{aligned}
$$

This rhyme is particularly remarkable given that it came many years before any link was postulated between avian and human influenza. Influenza is a variable entity: on the surface of the virus are many proteins, or antigens, each one of which can take a number of subtly different forms. It is these proteins that give the influenza virus its identity and allow the host's immune system to raise a specific and targeted defence against it. The two most important proteins are haemagglutinin $(\mathrm{H})$ and neuraminidase $(\mathrm{N})$ and

Chief Medical Officer for England it is their nature that determines the 'label' given to a specific type of influenza (such as 'H5N1'). Beneath the level of $\mathrm{H}$ and $\mathrm{N}$, and not reflected in the name, are a large number of lesser proteins, the character of which determines the precise strain of influenza virus.

From time to time, these proteins or antigens alter in character. Changes may be subtle when mutations of the minor proteins occur at the time of viral replication ('drift') or more dramatic where one of the major proteins is replaced by another ('shift'). Such dramatic shifts are most likely to take place when two distinct types of influenza are replicating in the same location at the same time and the opportunity for the mixing of genetic material arises.

It is when these proteins, and thus the very identity of the virus, alter that a significant new strain can come into existence. Such a strain then has the potential to infect large numbers of hosts - each with little or no defence against a viral strain that they have not previously encountered.

There are three distinct 'influenza scenarios' of relevance and it is vital that professionals and others are mindful of this: seasonal flu, avian flu and pandemic flu.

Seasonal flu occurs each winter. It can affect between 10 and $15 \%$ of the population. Most people experience one or two weeks of symptoms which are unpleasant but not life threatening, and they recover without requiring specific treatment. People who are either dependent on others or have reduced physiological reserve (including the very young, the very old and those with chronic diseases) are at risk of more serious illness. The strain of influenza likely to occur each winter in Europe is fairly predictable as changes from previous years are usually modest and the experience of the Southern hemisphere in the preceding six months can be informative: appropriate vaccines can therefore be prepared and are offered each autumn to those at risk of more serious illness. Vaccination is also offered to likely 'vectors' who have the potential to transmit influenza between those in the high risk group (such as healthcare workers and other carers). Anti-viral drugs can be used in specific circumstances to limit the impact of seasonal influenza in its early stages.

Avian flu, for the most part, is just that - a disease of birds. However, from time to time, the virus causing flu that predominantly affects one species is also capable of causing illness in others, including man. There are many examples of such outbreaks: a pregnant woman died in the 
USA in 1988 after contact with pigs during an outbreak of H1N1 swine fever, an adult presented with conjunctivitis caused by an H7N7 duck virus in the UK in 1995 and six people died in Hong Kong during 1997 as a result of H5N1 avian flu. The current series of human cases of $\mathrm{H} 5 \mathrm{~N} 1$ associated with the outbreak of avian influenza is one further example of disease that is capable, to a limited extent, of crossing species boundaries. To date (11 April 2006), there have been 109 human deaths since 2003 due to the current avian strain of $\mathrm{H} 5 \mathrm{~N} 1 .{ }^{1}$ In almost every case, the victim of the disease has had close contact with infected birds. There have been a limited number of well documented cases in which there is evidence to suggest that human to human transmission has occurred but at the time of writing, there is no evidence that the avian influenza virus has adapted to spread easily between humans.

The H5N1 strain of avian influenza currently circulating in poultry and other birds is highly pathogenic in this population. However, in spite of the extremely high burden of avian disease worldwide, the virus has to date, shown a very limited capacity to infect people. The concern is that this avian disease may transform into a strain of influenza that is better equipped to gain entry into the human body, and once there, cause significant disease against which humans have no natural immunity. Such transformation may occur in a number of ways: through mutation as the virus replicates in the bird population or more likely through genetic re-assortment in a host co-infected by two different influenza strains. Although such a host might be human, it could be porcine, where a human virus and avian influenza co-exist. From a public health perspective, the main concern in relation to the present outbreak of avian influenza is the high number of infected birds and therefore the very large pool of H5N1 virus present globally, thereby affording increased opportunity for mutation or reassortment into a form more transmissible between humans.

Pandemic influenza arises when a novel strain of flu, not previously encountered by the human immune system, emerges. Large outbreaks of influenza in the avian population may simply facilitate and hasten this emergence. Nature frequently follows cycles and it is likely that there have been influenza pandemics at regular intervals throughout history. In the recent past, these have been well-documented: the outbreak in 1918-1919 that caused the deaths of 40-50 million people (many more than the First World War), the outbreaks of Asian flu in 1957 and Hong Kong flu in 1968 that each caused a million additional deaths across the world. Some outbreaks have been caused by wholesale changes to the virus (or shift) whilst others have been due to more subtle changes (or drift), including the development of the Fujian strain of H3N2 from the Panama strain. Although these pandemics were similar in many ways, they also had their own specific characteristics in relation to the section of the population hit hardest, the proportion of exposed individuals who succumbed to disease and the duration of the outbreak.

Having been identified as a potential threat at the start of the year, the 1957 pandemic was first encountered in Britain in June. However, it was not until September that a large epidemic occurred, affecting predominantly children and their parents. There were a number of outbreaks over the summer: the impact of influenza on the Royal Air Force during this period was described by Walter Holland in the Lancet and provides an excellent illustration of the progress of the virus. ${ }^{2}$ In general though, older adults were largely spared until the winter, when a second wave (with a higher mortality) struck.

The appearance of the 1968 pandemic strain in the UK was relatively innocuous, with modest outbreaks to start with (unlike its more dramatic arrival in the United States). It was not until the following winter that an explosive epidemic took hold.

An influenza pandemic will arrive at some point. It is impossible to predict with certainty when this will happen but there are three conditions necessary for it to occur: the emergence of a new influenza strain to which large numbers of the population have not been exposed; the emergence of a strain that is readily transmitted from person to person; and the emergence of a strain that is capable of causing human illness.

The scale and impact of an influenza pandemic in the UK is impossible to predict with absolute accuracy before the particular influenza strain has emerged and its early behaviour has been observed. Any outbreak of an infectious disease has a clinical attack rate, describing the proportion of an exposed population who go on to develop illness, and a case fatality rate reflecting the death rate in those who become ill. Estimates vary significantly but based on the assumption of a clinical attack rate of $25 \%$ and a case fatality rate slightly higher than 1 in 300 , the UK could expect to see around 50000 excess deaths.

While modern technology has given us the ability to predict and explain pandemics, we cannot prevent them. However, there are a number of things that we can do in an attempt to reduce their impact. These measures can be broadly categorized as the use of anti-virals, vaccination and non-pharmaceutical public health measures. Each one of these categories has been thoroughly examined by the UK Government and each plays an important role in the welldeveloped UK Influenza pandemic contingency plan, published jointly by the UK Health Departments during $2005 .^{3}$

Anti-viral drugs hinder the replication of the influenza virus. In the context of seasonal flu, they can shorten illness by a day and importantly, halve hospitalizations. They are 
already offered to those at risk of more serious illness following infection with seasonal flu. In order to be effective, anti-virals must be taken within 48 hours of the onset of symptoms and the sooner the better. The UK has taken the precautionary step of building up a stockpile of oseltamivir (Tamiflu) for use in the course of a pandemic. The stockpile consists of 14.6 million courses, sufficient for a quarter of the population (based on a clinical attack rate of $25 \%)$.

Vaccination is an equally important aspect of the UK's defence strategy. There are two schools of thought, the first that one should purchase large quantities of vaccine against the current strain of avian flu from which any pandemic is likely (although by no means certain) to evolve. Indeed, one could potentially vaccinate against the $\mathrm{H} 5$ antigen alone (strengthened through the use of an adjuvant) in advance of a pandemic. Such a strategy may offer a degree of protection against a pandemic strain, sufficient to have a considerable effect in diminishing the impact of a pandemic. However, if the pandemic strain was very different, it could conceivably offer no protection at all. The second school of thought is that one cannot produce a vaccine that is certain to be reliable and effective until such a time as the precise pandemic virus has emerged (and has been subsequently identified). The view of the UK Government is that both strategies need to be pursued urgently with the scientific community and manufacturers. Vaccine production is not an instantaneous process and even following the precise characterization of a virus, the manufacturing and testing stages of vaccine development are critical.

Arrangements are already in place for the accelerated licensing of vaccines through the European Medicines Evaluation Agency. Companies are submitting 'mock-up' files before a pandemic, of the products that they anticipate producing once the pandemic strain is known. This approach means that much of the manufacturing and safety data can be evaluated in advance and a rapid licence variation can be issued to accommodate the strain change to the pandemic strain. The length of time required between isolation of the relevant strain of influenza and delivery of vaccine has been condensed as much as is possible, but nevertheless it will still take around four months after pandemic viral isolation before a vaccine starts to become available. The pandemic specific vaccine is therefore unlikely to be available during a 'first wave' of a pandemic.

Plans have been formulated such that large supplies of the relevant pandemic vaccine are available to the UK population as soon as manufacturers can produce it. There will be intense international demand for vaccine and countries that do not have arrangements in place face the likelihood that they will not have any vaccine at all. The UK is negotiating 'sleeping contracts' with large-scale vaccine producers such that manufacturing capacity is guaranteed and 120 million doses of specific vaccine will be available for the whole population as soon as possible.

The UK Government has also acquired a supply of 3.5 million doses of 'adjuvanted' $\mathrm{H} 5 \mathrm{~N} 1$ vaccine. This will be used for research purposes and if the risk of an H5N1derived pandemic increases markedly, it would be available to be offered to frontline NHS staff. This group has been chosen for their personal protection (so that the NHS workforce remains able to deliver services) but also to reduce their ability to inadvertently transmit the virus between their patients and other contacts.

Other public health precautions will also be taken when a pandemic arises but none is failsafe. Modelling work has demonstrated that a ban on international travel, even if $99 \%$ effective, would only delay the global spread of a pandemic by a few weeks. Against this possible gain, there would be a very marked impact upon international trade, in a world that is now very much interdependent. Other measures that would be considered as part of the contingency plan include the closure of schools and the possible cancellation of large public events (such as football matches and pop concerts).

To date, there has been no definitive evidence of transmission of influenza to humans by eating meat from an infected animal. There is evidence that the slaughter and handling of ill or dead birds does pose a risk and these activities should be avoided wherever possible. Although eating contaminated animal products is not known to be dangerous, the European Food Safety Authority, the European Centre for Disease Prevention and Control, and the World Health Organization offer precautionary advice that is supported by the UK Government: heating the influenza virus to 70 degrees celsius kills it and therefore eggs and poultry should be cooked appropriately. ${ }^{4,5}$

Technology offers many welcome opportunities to reduce the impact of an influenza pandemic through the interventions described. However, along with these opportunities comes a degree of uncertainty and fear. In an age of information, the public understandably demands details of any threat. At a time when the human race has come to regard itself as its own master in many spheres, the power and force of nature can be difficult to comprehend and accept. Just as another tsunami will one day happen, so too will a pandemic come. We can design warning systems and develop interventions to modify the impact but we cannot prevent these natural phenomena. It is vital that in a sea of information, journalists behave responsibly and misinformation (either inadvertent or intentional) is minimized.

Attempting to ensure that information is accurate is one vital pillar of the UK's pandemic flu planning. In the last quarter of 2005, I commissioned a study to assess media coverage of the issue and the impact of my efforts to promote accuracy. Over this period, the British media 
(print, television, radio and internet) collectively generated over 1.4 billion 'weighted opportunities to see' information relating to the flu pandemic. This is equivalent to 30 exposures to information per adult. 250 million 'weighted opportunities' is regarded as the yardstick for a 'megastory'. More detailed analysis demonstrated that the key messages of the contingency plan were accurately and positively portrayed in the media.

The issue of pandemic influenza will be with us for some years to come. It is vital that we embrace the opportunity to better prepare ourselves for the arrival of human illness but equally important that we maintain an appropriate perspective on this most natural of phenomena.

\section{REFERENCES}

1 [http://www.who.int]

2 Holland W. A clinical study of influenza in the Royal Air Force. Lancet 1957;273:840-1

3 UK Health Departments' Influenza pandemic contingency plan (October 2005 edition) [http://www.dh.gov.uk]

4 [http://www.efsa.eu.int]

5 [http://www.ecdc.eu.int] 